angiogenesis. The aim of the present study was therefore to investigate the specific influence of Nox NADPH oxidases on PiPS-derived ECs.

Methods Human neonatal lung fibroblasts were subjected to direct reprogramming by viral transduction with the transcription factors, OCT4, SOX2, Klf4 and c-Myc, for 4 days to generate PiPS cells, which were then seeded on mouse collagen IV in EGM-2 media for a further 6 days to induce EC differentiation.

Results PiPs cells were defined by induced expression of KDR and CD34, whilst PiPS-ECs were characterised by expression of endothelial markers, CD144 and CD31. Expression of NADPH oxidase isoforms, Nox1, Nox2 and Nox4 (as assessed by real-time RT-PCR) was progressively induced during reprogramming and EC differentiation (e.g. Nox4 mRNA: fibroblasts, $2.1 \pm 0.2$; PiPS, $36 \pm 4$; PiPS-ECs, $133 \pm 29$ arbitrary units; $\mathrm{n}=3, \mathrm{P}<0.001$ ), with comparable levels observed in PiPS-ECs (Nox1 mRNA, $126 \pm 20$; Nox2 mRNA, $82 \pm 20$; Nox4 mRNA, $133 \pm 29$ arbitrary units; $n$ $=3, \mathrm{P}=\mathrm{NS}$ ). Notably, mRNA expression of Nox4, which has been previously linked with angiogenesis, was markedly increased during hypoxia (1\% oxygen) in PiPS-ECs compared with the other two isoforms (Nox1, $2.89 \pm 0.26$; Nox2, 1.52 \pm 0.06 ; Nox $4,8.04 \pm 0.76$ arbitrary units; $\mathrm{n}=3, \mathrm{P}<$ 0.001).

Conclusions These data suggest that NADPH oxidases, particularly Nox4, may be important in mediating angiogenic function in PiPS-ECs. Future work will investigate whether genetic modification of Nox4 can influence reprogramming and differentiation of PiPS-ECs and consequently their ability to promote angiogenesis in response to experimental ischemia.

\section{B CELL-SPECIFIC FC GAMMA RECEPTOR IIB OVER- EXPRESSION ENHANCES ATHEROSCLEROSIS BY INHIBITING B1 CELLS}

Andrew Sage*, Deirdre Murphy, Leanne Masters, Lauren Kitt, Reem Assil, Ziad Mallat. University of Cambridge; *Presenting Author

\subsection{6/heartjnl-2016-309890.206}

Antibodies binding oxidised epitopes on apoptotic cells and oxidised forms of LDL form an important protective barrier slowing the development of atherosclerosis. However, not all $\mathrm{B}$ cell functions or subsets are protective since adaptive B2 cell depletion is atheroprotective and autoimmunity is associated with accelerated atherosclerosis. The inhibitory IgG receptor $\mathrm{FcI}^{3} \mathrm{RIIb}$ is the only IgG receptor found on $\mathrm{B}$ cells and also counteracts pro-inflammatory signalling from activating $\mathrm{FcI}^{3}$ receptors in macrophages and dendritic cells. Studies on $\mathrm{FcI}^{3} \mathrm{RIIb}$ knockout mice demonstrate significant regulation of atherosclerosis but contrasting effects depending on the genetic background, suggesting the need for alternative approaches. We have analysed the effects of B cell-specific over-expression of $\mathrm{Fc}^{3} \mathrm{RIIb}$ using a previously characterised transgenic mouse strain. $\mathrm{FCl}^{3} \mathrm{RIIb}$ over-expression was confirmed to be specific for $\mathrm{B}$ cells and was equal between genders. There was only minor impacts on mature $\mathrm{B}$ cell levels, but $\mathrm{Fc}^{3} \mathrm{RIIb}$ overexpression significantly attenuated plasma cell levels and serum antibody titres. Female, but not male, Fcîl ${ }^{3} \mathrm{RIIb}-\mathrm{B}$ cell transgenic mice on the $\mathrm{ApoE}^{-/-}$background develop significantly enhanced atherosclerosis after 6 weeks high fat diet compared to non-transgenic littermates. Females but not males had significantly decreased oxidised epitope-specific IgM levels and $\mathrm{B} 1 \mathrm{a}$ cells in spleen and peritoneal lavage. In vitro, B1 cells from female $\mathrm{ApoE}^{-/-}$mice were more susceptible to $\mathrm{Fc}^{3} \mathrm{RIIb}-$ induced apoptosis. Overall, our study highlights a potential difference in B1 cell biology between males and females, and supports the investigation of gender specific effects of autoimmune-linked $\mathrm{Fc}^{3} \mathrm{RIIb}$ polymorphisms on human cardiovascular disease.

\section{THE INTERPLAY BETWEEN THE RENIN ANGIOTENSIN SYSTEM AND PACING POSTCONDITIONING INDUCED CARDIAC PROTECTION}

Fawzi Babiker*, Aishah Al-Jarallah, Shaji Joseph. Kuwait University; *Presenting Author

\subsection{6/heartjnl-2016-309890.207}

Background Accumulating evidence suggests a cardioprotective role of pacing postconditioning (PPC). The interaction between RAS and PPC induced cardiac protection is however not clearly understood. The role of angiotensin converting enzyme (ACE), Angtiosin II (Ang II) and angiotensin receptor 1 (AT1) remains to be identified.

Objective The objective of this study was therefore to investigate the role of ACE-Ang II-AT1 axes of RAS in the protective effects of PPC.

Methods The role of RAS was tested by infusion of Ang II, chymostatin (inhibitor of locally produced Ang II), ACE blocker (captopril) and AT1 antagonist (irbesartan). Hemodynamics data was computed digitally and infarct size was determined using 2,3,5-Triphenyltetrazolium chloride (TTC) staining and by measuring creatine kinase $(\mathrm{CK})$ and lactate dehydrogenase (LDH) levels.

Results In comparison to hearts subjected to $\mathrm{I} / \mathrm{R}$ injury or untreated control hearts, PPC significantly $(\mathrm{P}<0.001)$ improved cardiac hemodynamics and reduced infarct size and cardiac enzymes. Systemic infusions of Ang II did not affect I/ $\mathrm{R}$ injury or PPC mediated protection. Nonetheless inhibition of endogenously synthesised Ang II protected against I/R induced cardiac damage and did not block or augment the protective effects of PPC. The administration of AT1 antagonist did not alleviate $\mathrm{I} / \mathrm{R}$ induced damage. Interestingly it abrogated PPC induced cardiac protection. Finally, PPC induced protection and blockade of locally produced Ang II involved enhanced activation of ERK1/2 and Akt.

Conclusions This study demonstrate a novel interaction between PPC mediated cardiac protection and ACE-Ang IIAT1 axes of the RAS in which locally produced Ang II appears to play a significant role in PPC mediated protection while systemically produced Ang II appears to be dispensable. Moreover this interactions appears to involve alterations in the activation state of downstream kinases including ERK1/2 and Akt.

Acknowledgement This study is supported by grant number MY 02/10 from Research Administration, Kuwait University, Kuwait. 